\title{
2020 y la crisis de Brasil como crisis de nuestra América: hacia una defensa del estado de bienestar en América Latina y el Caribe, más allá del Estado nacional
}

\author{
Recibido: 7 de agosto de 2020 • Aprobado: 10 de septiembre de 2020 \\ https://doi.org/10.22395/ojum.v20n42al
}

David Gomes

Universidad Federal de Minas Gerais (UFMG), Belo Horizonte, Brasil

davidflgomes@yahoo.com

https://orcid.org/0000-0003-0948-5860

\section{RESUMEN}

Este artículo tiene por objetivo discutir la crisis actual en Brasil como una crisis de Latinoamérica y el Caribe. En ese sentido, traslada metodológicamente el debate desde la bibliografía predominante sobre las crisis democráticas hacia una teoría de la sociedad de tres niveles. En su primera parte, se presenta y desarrolla de forma sintética la idea de una teoría de la sociedad de tres niveles. En la secuencia, en la segunda parte son discutidos los elementos principales para la comprensión de la crisis brasileña contemporánea. Por fin, en las conclusiones es planteada la tesis de que la crisis de Brasil debe ser comprendida como una crisis de nuestra América y que, por lo tanto, la defensa del Estado de bienestar en Latinoamérica y el Caribe debe ser una defensa más allá del Estado nacional. Así, los resultados que se exponen indican la necesidad de una mayor aproximación entre los distintos países de América Latina y del Caribe como forma de garantizar una mejor protección a la democracia y al Estado de bienestar en la región.

Palabras clave: crisis democrática; estado de bienestar; capitalismo; Brasil; América Latina; Caribe. 


\title{
2020 and the Brazilian Crisis as a Crisis of our America: Towards a Defense of the Welfare State in Latin America and the Caribbean Beyond the National State
}

\begin{abstract}
This article's goal is to discuss the current Brazilian crisis as a Latin American and the Caribbean crisis. Thus, this article methodologically translates the debate of the predominant bibliography upon democratic crisis towards a theory of societies in three levels. The first part presents and synthetically develops the idea of a theory of society in three levels. The second part discusses the main elements for the comprehension of the current Brazilin crisis. Lastly, the article states in its conclusions that the current Brazilian crisis must be understood as a crisis of our America and, thus, the defense of the welfare state in Latin America and the Caribbean must go beyond the nation-state. Thereby, the results indicate the need for greater contact among the Latin American and the Caribbean countries as a way for ensuring better protection of democracy and the welfare state in the region.
\end{abstract}

Keywords: democratic crisis; welfare state; capitalism; Brazil; Latin America; Caribbean. 


\section{INTRODUCCIÓN}

Este artículo proviene del proyecto de investigación Derecho, Modernidad y Capitalismo, que coordino hace tres años en la Facultad de Derecho de la Universidad Federal de Minas Gerais, en Brasil, en las carreras de grado y de posgrado. Agradezco a Ana Clara Presciliano, Henrique Pereira de Queiroz, João Pedro Lopes Fernandes, Maria Carolina Fernandes Oliveira y Marina Leite de Moura e Souza por sus comentarios a la primera versión del texto. Agradezco también al profesor Ramiro Chimuris — de la Udelar en Uruguay — y al profesor Juarez Guimarães — de la UFMG en Brasil— por su cuidadosa lectura y sus preciosas sugerencias. Y agradezco de la misma manera al profesor Gonzalo Scivoletto — de la UNCuyo, en Argentina- y a su equipo de investigación por el rico conversatorio que han organizado sobre el tema del artículo.

Alrededor del mundo se discute hoy la cuestión de la crisis de la democracia y de su forma institucional contemporánea: el Estado democrático de derecho. Por lo menos en Brasil, esa discusión ocurre fundamentalmente sobre la base de textos escritos por autoras y autores (Castells, 2018; Levitsky y Ziblatt, 2018; Mounk, 2019; Runciman, 2018) de otros contextos sociales y académicos muy distintos de los nuestros en América Latina y el Caribe (Sevaybricker, 2019). Así, en general, se olvida el aprendizaje social y teórico que, por ejemplo, logramos duramente con los horrores de la experiencia histórica de nuestras dictaduras en el siglo XX. Ese olvido no puede empero generar sorpresa: uno de los rasgos comunes entre aquellas autoras, aquellos autores y quienes aplican sus teorías, sin mediaciones, para comprender nuestra situación actual suele ser un profundo déficit sociológico.

Sin duda, también podemos aprender algo de las experiencias de otras sociedades y de las correspondientes elaboraciones teóricas. Pero es demasiado ingenuo presuponer que podemos aprender algo sobre nosotros mismos de esas experiencias y teorizaciones ajenas solamente.

En ese sentido, a mí me gustaría enfocar el problema de la crisis de la democracia en Brasil y en América Latina y el Caribe desde una mirada distinta, desarrollada en el seno de una teoría de la sociedad de tres niveles, y tomarla fundamentalmente como una crisis del estado de bienestar.

\section{UNA TEORÍA DE LA SOCIEDAD DE TRES NIVELES}

\subsection{El primer nivel}

En un primer nivel, es menester reconocer que los procesos de modernización han forzado una homogeneización inédita. Esa homogeneización puede ser criticada y lamentada desde un punto de vista normativo, pero no puede ser negada desde un punto de vista descriptivo. Como consecuencia, es posible indicar patrones 
comunes que definen a las sociedades modernas independientemente de su ubicación geográfica o geopolítica.

Si se tienen en consideración los principales procesos que mantienen las sociedades modernas integradas, entonces se ve con claridad que dos dinámicas rivalizan en esa tarea social integradora: la dinámica generada desde la economía por los imperativos de autovalorización del capital (Marx, 2011, 2013, 2014, 2015) y la dinámica engendrada por las expectativas normativas propias de una forma sociocultural de vida. En esta última la individuación de sus miembros es al mismo tiempo una socialización que transcurre sobre todo en medio del lenguaje (Habermas, 2010a, 2010b).

Los imperativos de autovalorización del capital se manifiestan sistémicamente como una dominación anónima que, empero, se va encarnando en normas, instituciones y orientaciones de acciones individuales y colectivas. A su vez, las expectativas normativas emergen en los contextos intersubjetivos de vida cotidiana y desde ahí van a enfrentarse con aquellos imperativos sistémicos en las mismas normas, instituciones y orientaciones de acciones.

Las sociedades modernas pueden, por lo tanto, ser definidas como una tensión constante entre imperativos sistémicos, derivados de la presión del capital por su autovalorización, y expectativas normativas que, arraigadas antropológicamente en una especie constitutivamente marcada por la adquisición evolutiva del lenguaje, se van liberando más intensamente solo en la misma modernidad (Habermas, 2010b; Gomes, 2019).

Así, en una sociedad moderna cualquiera, sea en Europa o en Asia, hay una historia de luchas sociales que no encuentra un equivalente premoderno en el contenido normativo de sus reivindicaciones. De otro lado, hay problemas similares que remiten en último grado a la organización capitalista de la producción y de la circulación económica, por ejemplo, las tendencias cíclicas de crisis económicas y los efectos en general de la ley de la caída tendencial de la tasa de ganancia (Marx, 2015, pp. 249-306).

\subsection{El segundo nivel}

No obstante, si hay rasgos comunes entre sociedades modernas ubicadas en regiones diferentes del mundo, hay también distinciones fundamentales. Así, en un segundo nivel de reflexión es menester tener en cuenta que la modernidad se ha desarrollado desde sus comienzos con una doble cara: su cara oculta ha sido y sigue siendo la colonialidad (Dussel, 1994, 2005; Mignolo, 2003; Quijano, 1992), es decir, la división del mundo por un clivaje que constituye, de un solo golpe, la unidad de la modernidad y su escisión entre Norte y Sur o, en otro lenguaje conceptual, entre centro y periferia. 
Con ese paso metodológico no se cambia la definición de modernidad y de sociedad moderna, sino que esa definición sufre un proceso de refracción: la sociedad moderna se caracteriza fundamentalmente en su arquitectura formal como una tensión constante entre imperativos sistémicos del capitalismo y expectativas normativas de una vida emancipada, pero esa tensión se desarrolla tendencialmente de una manera en el Norte y de otra manera en el Sur. Esto quiere decir que la tensión se refracta: ella sigue siendo la misma tensión, pero cambia la dirección de su camino al cambiar el medio en el que se encuentra.

Las expectativas normativas de la modernidad alcanzan un amplio rango de ámbitos de vida, desde la formación de la subjetividad hasta demandas morales muy abstractas. El punto que más interesa aquí es el de las expectativas normativas que se refieren a la autodeterminación política de una sociedad. Si se parte de esa perspectiva, la tensión definidora de la modernidad puede ser presentada en una versión más estrecha: como una tensión entre capitalismo y democracia.

Si se piensa entonces en la refracción de esa tensión entre capitalismo y democracia, se puede afirmar que la tensión es la misma en el Norte y en el Sur, pero que su desarrollo tendencial es distinto en una y en otra ubicación geopolítica. En el Norte, la tendencia es la de una relativa estabilización entre capitalismo y democracia a lo largo del tiempo, mientras que en el Sur la tendencia es la de una constante inestabilidad de la relación entre capitalismo y democracia. Así lo concluye, por ejemplo, en sus propios términos Wanderley Guilherme dos Santos, más directamente sobre el caso de Brasil en 1962, o sea, poco antes del golpe dictatorial de 1964: "la amenaza de golpe no es un fenómeno reciente en la sociedad brasileña; por el contrario, se manifiesta todas las veces que el movimiento de ascenso popular retoma impulso" (Santos, 1962, p. 2).

Pero esas tendencias diferentes en el Norte y en el Sur no solo se yuxtaponen, sino que se explican recíprocamente. La inestabilidad histórica de la relación entre democracia y capitalismo en el Sur no es simplemente el opuesto de su estabilidad en el Norte: más bien, es su complemento necesario. En las palabras de Ruy Mauro Marini (1971): "La historia del subdesarrollo latinoamericano es la historia del desarrollo del sistema capitalista mundial" (p. 3).

De un lado, la posibilidad de una explicación estructural referida al modo de producción capitalista le da a la inestabilidad de la relación entre capitalismo y democracia en el Sur el carácter de una tendencia, es decir, algo más que una contingencia históricamente verificable. Sin embargo, de otro lado, esa explicación estructural es insuficiente para aclarar los complejos procesos en que esa tendencia adquiere concreción histórica. Por ello, la teoría de la dependencia latinoamericana no ha sido nunca solo una teoría económica de acento estructuralista: la dependencia tiene múltiples facetas (Fernandes, 1973). 
Una de sus facetas con mayores consecuencias prácticas es el comportamiento — siempre enfocado como tendencial, no como mecánicamente determinado- de las élites políticas en los países del Sur (Buitrago, 2014). Herederas en regla de las antiguas élites coloniales, su relación con el antiguo universo metropolitano suele ser bastante profunda, así que incluso la configuración de sus identidades — su autocomprensión, su referencia cultural, sus objetivos y sueños de vida - revela un vínculo simbólico mucho más fuerte con el Norte que con sus propias sociedades en el Sur.

En la medida en que ese elemento valorativo y axiológico viene generalmente imbricado con intereses económicos de elevado montante, no es difícil comprender el ambiguo rol que esas élites se disponen a jugar: paradojalmente, como élites subordinadas, tienden a aceptar un lugar secundario en el escenario de las relaciones internacionales para que puedan mantener, en el escenario interno de sus respectivos países, la ilusión de su vinculación al Norte, al centro del mundo. De ahí se deriva el abismo entre ellas y el resto de las poblaciones locales.

Dos efectos concretos advienen de la actuación política local de esas élites subordinadas: la facilidad con que intereses económicos extranjeros se imponen en la agenda y la ausencia de proyectos nacionales de desarrollo e inclusión, o, cuando los hay, la crítica destructiva que se les opone, lo que suele ocurrir con la reducción de todo intento de un proyecto exitoso de Estado de bienestar bajo la categoría de "populismo" (Ferreira, 2010; D'eramos, 2013).

\subsection{El tercer nivel}

Esa primera refracción geopolítica desplaza entonces el análisis desde un primer nivel de abstracción que tiene en consideración características globales de la sociedad moderna hacia un segundo nivel en el que se saca a luz el clivaje colonial y la escisión del mundo moderno entre Norte y Sur, entre centro y periferia.

Pero todavía ese paso metodológico, si no es entendido adecuadamente, puede ofrecer una visión demasiado estructural-determinista de la historia y de sus posibilidades. Por consiguiente, es menester una segunda refracción geopolítica hacia un tercer nivel de reflexión en el que la urdidura de las historias locales — por ejemplo, las tramas de las historias sociales de una nación o un pueblo- vengan al centro del análisis.

Las sociedades de Brasil, Argentina o Uruguay son sociedades modernas que igualmente se ubican geopolíticamente en el Sur global, en la periferia del sistemamundo moderno-capitalista (Quijano y Wallerstein, 1992). Sin embargo, si uno se limita a esa ubicación común y a sus consecuencias estructurales como factor de explicación, no puede comprender las diferentes trayectorias que tales sociedades han seguido, a pesar de todas las similitudes. 
En lo que atañe específicamente a Brasil y más directamente a sus sucesos políticos recientes, tres factores fundamentales importan acá: uno, de más larga duración, es la herencia del sistema esclavista; otro, también arraigado desde hace muchos años, es la equiparación naturalizada entre Estado y corrupción; finalmente, el tercero es la realización precaria e interrumpida de un proceso de justicia transicional antes de que se pudieran generar los impactos sociales esperados.

\section{LA CRISIS EN BRASIL: ELEMENTOS PARA SU COMPRENSIÓN}

\subsection{Elementos compartidos por otras sociedades del mundo}

En la moldura de una teoría social de tres niveles, presentada sucintamente en el anterior capítulo, se puede llevar adelante el esfuerzo de comprensión de lo que sucedió en Brasil en el año 2020. En primer lugar, seguramente hay elementos de la crisis brasileña que son comunes a otras sociedades del globo, y que se van a manifestar en regímenes autoritarios con diferentes grados y perfiles.

Me gustaría mencionar tres de esos elementos. El primero es el agotamiento de la ilusión del crecimiento económico continuado y sin crisis. Esta ilusión ha ganado una fuerza increíble desde los llamados "treinta gloriosos" en Europa, esto es, las tres décadas luego de la Segunda Guerra mundial. Desde entonces, se tienen nuevamente las viejas crisis cíclicas y la última de las grandes crisis que ha empezado en los años de 2007 y 2008, y todavía produce sus efectos.

El segundo elemento es la narrativa neoliberal. Esta pudo apoyarse en la ilusión objetiva del crecimiento económico continuado sin crisis para convertirse, sobre todo después de la desintegración de la Unión Soviética, en una narrativa hegemónica. Esta narrativa se esfuerza en reducir toda la complejidad de la vida a interacciones simples entre sujetos aislados e impulsados solo por intereses.

El tercer elemento tiene un alcance antropológico más profundo. Se refiere a la pérdida de la centralidad de la escrita — del lenguaje escrito — en las comunicaciones sociales como un todo. Esta es una hipótesis explicativa menos intuitiva y requiere un trabajo argumentativo que he intentado desarrollar en otro lugar (Gomes, 2020). En una forma muy sintética, puede decirse que se ha establecido en la modernidad una constelación específica entre sociedad, razón, verdad, derecho, democracia y emancipación. Esa constelación, a su vez, ha sido estabilizada gracias al rol desempeñado en la modernidad por la comunicación escrita que, potenciada por la invención de la prensa, pudo ubicarse sociológicamente en el centro de gravitación de las comunicaciones sociales en general, incluso en sociedades con poblaciones predominantemente analfabetas (Gomes, 2020, 2019, pp. 73-131). Entre fines del siglo XX y comienzos del siglo XXI esa situación ha cambiado: la comunicación escrita ha sido desplazada desde el centro de gravitación de las comunicaciones sociales como un todo, donde la propia idea de un centro de gravitación de las comunicaciones parece haber 
perdido su lastre sociológico. En la medida en que la centralidad del lenguaje escrito ha sido imprescindible para que aquella constelación moderna se deviniera estable, la pérdida de la centralidad de la escrita la pone en jaque. Por esa razón, las crisis contemporáneas de la democracia vienen acompañadas de una crisis de legitimación social de la ciencia como portavoz institucional de la verdad: democracia y ciencia son dos elementos de una misma constelación que ahora se vuelve inestable. Las noticias falsas, de un lado, el negacionismo científico, de otro, y la relación entre estas dos son los síntomas más claros de la debilidad actual de la constelación entera.

\subsection{Elementos especificos del Sur global y de Latinoamérica}

El cierre de los "treinta gloriosos" ha fomentado en el Norte una crítica feroz al estado de bienestar. Así, desde la década de 1970 esa forma institucional vive una crisis ininterrumpida. Si esa crisis echa sus raíces en la estructura profunda de un modelo que intenta arreglar institucionalmente la compatibilidad entre capitalismo y democracia (Streeck, 2012), no se podría esperar que la misma crisis no viniera a alcanzar los proyectos de bienestar que se lograron implantar en Latinoamérica al comienzo del siglo XXI.

Pero si la crisis objetiva del estado de bienestar en el Norte en regla no ha sido acompañada de una amenaza efectiva al Estado de derecho - en términos más precisos, no ha desencadenado rupturas institucionales y golpes de Estado-, no ocurre lo mismo en América Latina y el Caribe. La presente situación de Ecuador, Brasil y, más que todo, de Bolivia nos hace recordar una vez más la fuerza práctica de la mencionada tendencia hacia una constante inestabilidad en la relación entre capitalismo y democracia en el Sur. Y no es producto del azar que incluso los actos más directamente antidemocráticos se intentan justificar con supuestos argumentos de "crítica al populismo".

Además de esa tendencia estructural que se puede verificar en América Latina y el Caribe, en África o en Asia, y que hace acercarse la crisis del Estado de bienestar a la crisis del Estado de derecho como un todo, otro elemento es imprescindible en el nivel teórico - que luego de una primera refracción geopolítica deja tras de sí las formulaciones de alcance global — para comprender el caso de Brasil en 2020 o, más bien, desde 2018. No se trata, empero, de un elemento que pueda ser presentado sin más como tendencia característica del Sur global, sino que puede ser afirmado como un rasgo muy propio de América Latina. Así, un tema debe ser enfocado entre la primera y la segunda refracción geopolítica, entre el segundo y el tercero de los niveles metodológicos fundamentales de la teoría social esbozada precedentemente. Estoy refiriéndome a la religión -y más específicamente al cristianismo - como dadora de sentido a la vida cotidiana.

Este es también un elemento que requiere una explicación con más detalles, y que todavía no he podido presentar. En su expresión sintética, la hipótesis 
correspondiente es la siguiente: luego del Concilio Vaticano II, el cristianismo en Latinoamérica ha sido fuertemente influido por la teología de la liberación (Dussel, 1999; Vilela, 2013), tanto en la iglesia católica como en las iglesias protestantes. Esa comprensión teológica ha sufrido resistencia dentro de las iglesias mismas, como lo demuestran los sucesos con el Vaticano. Así, ella nunca ha devenido una interpretación oficial del cristianismo, pero su fuerte presencia en la práctica del cristianismo latinoamericano ha generado algo como un sentido común religioso que sobrepasaba las fronteras de la teología de la liberación y de sus encarnaciones institucionales, como las comunidades eclesiales de base. A ese trasfondo de un sentido de religiosidad compartido lo llamo narrativa teológico-comunal. En esta, la relación con Dios es interpretada como manifiesta fundamentalmente en las relaciones con las personas prójimas — la idea de "amor al prójimo"—, sobre todo con aquellas que más sufren. Por lo tanto, lo sagrado tiene una dimensión predominantemente intersubjetiva, y la consiguiente idea de una responsabilidad compartida por las vidas como un todo en la sociedad ofrece el lastre para una comprensión de mundo a la que se puede acomodar sin mayores dificultades el proyecto democrático redistributivo del estado de bienestar.

Pero ese escenario ha cambiado desde fines del siglo XX y comienzos del siglo XXI. La narrativa teológico-comunal ha sido paulatinamente desplazada por un nuevo sentido común religioso: la narrativa teológico-sacramental. Su expresión más evidente son las iglesias neopentecostales (Panotto, 2013), y su forma doctrinaria más conocida es la teología de la prosperidad. Sin embargo, como un trasfondo de sentido de religiosidad compartido, esta sobrepasa el ámbito neopentecostal y antecede, desde el punto de vista de sus condiciones sociológicas de génesis, a la difusión de la teología de la prosperidad.

El rasgo característico de esa narrativa teológico-sacramental es que lo sagrado se piensa como si estuviera ubicado primordialmente en objetos sacros enredados en rituales sacros, esto es, ubicado en sacramentos en un sentido amplio para el término. De ese rasgo fundamental resultan algunas consecuencias importantes en el seno del sentido compartido de religiosidad que alrededor de él se forma. Dos de esas consecuencias merecen ser destacadas.

En primer lugar, la relación con Dios se traslada desde la relación intersubjetiva con otras personas - sobre todo con las que más sufren - hacia la relación individual con el sacramento mismo. De esta manera, la propuesta redistributiva del estado de bienestar e incluso la idea de una responsabilidad social común, sin la cual la democracia no es posible, pierden lastre en el sentido de comprensión cotidiano de las acciones de la vida. Al revés, ahora las ideas individualistas neoliberales se ven corroboradas en prácticas cotidianas tomadas como fundamentales por sus propios participantes, es decir, el paradójico proyecto no-social de sociedad se erige sobre la base socialmente insostenible de un egoísmo generalizado. 
En segundo lugar, como los sacramentos mismos no hablan se hace necesaria una interpretación que les asigne a ellos una significación. Así, la figura sacerdotal vuelve a tener una relevancia que se había debilitado en el contexto de la narrativa teológico-comunal. Esa relevancia no tiene más, empero, el rasgo propiamente jerárquico que depende de mediaciones institucionales, típico de la noción de autoridad que se ha desarrollado a lo largo de los siglos en la Iglesia católica romana (Arendt, 2000): ahora ella se manifiesta como relación directa, no mediada, entre la masa de los fieles y la figura sacerdotal que ante ellos presenta y representa el sacramento y que a su vez presentifica el propio Dios. Con esa estructura - una relación inmediata con una figura sacerdotal que en última instancia representa la voluntad incuestionable de Dios-- dicha relación prefigura relaciones sociales autoritarias y personalidades agudamente susceptibles a estímulos autoritarios en un sentido contemporáneo de autoritarismo, esto es, en un sentido que no tiene que ver con aquella noción de autoridad presentada por Arendt. En el seno de la filosofía política de Arendt (2000, 2006), el autoritarismo contemporáneo podría ser comprendido, más bien, como un tipo de gobierno al cual le hace falta precisamente autoridad y, por ello mismo, es menester utilizar otros recursos para mantener la capacidad de gobernar, incluso la violencia. En este sentido, una vez más, ese nuevo sentido compartido de religiosidad amenaza las bases de la democracia.

\subsection{Elementos de la especifica historia social brasileña}

En 2013, imágenes de las protestas en Brasil circularon en todo el mundo. Las múltiples demandas que salieron a las calles, muchas de ellas contrarias unas a las otras, son síntomas tajantes de lo que estaba por ocurrir: la entrada en crisis del proyecto de estado de bienestar que, aunque con limitaciones, se había establecido en el país desde los comienzos de la década de 2000.

La crisis objetiva del intento de conciliación entre capitalismo y democracia exigía, como toda crisis, una decisión (Habermas, 2013, pp. 375-389). Pero, como crisis objetiva relacionada con la crisis global del capitalismo en su refracción en el Sur, no indicaba una dirección a seguir. La cuestión que se impone entonces es: ¿cómo de esa crisis objetiva, abierta por definición a distintas decisiones, se ha pasado al escenario específico que se verifica en el Brasil del año 2020?

La respuesta empieza por llamar la atención para la imbricación entre la narrativa neoliberal y la narrativa teológico-sacramental. No se trata de preguntar por relaciones de causalidad entre una y otra, sino de comprender cómo han formado juntamente una constelación hegemónica en la tarea de asignar sentido a las acciones y decisiones de la vida, desde la más elementales hacia las más complejas. Así, ya en el trasfondo de un horizonte de sentido de mundo compartido se fue consolidando una imagen de la sociedad, las personas y los proyectos de vida que esas personas desarrollan en sociedad, en la cual 
no hay lugar para la responsabilidad común por la vida social y menos aún para la responsabilidad recíproca por las vidas individuales y colectivas dentro de esa sociedad.

Si con esa imagen consolidada en el trasfondo del horizonte de sentido socialmente compartido ya quedaba difícil que la decisión delante de la crisis fuera en un sentido del fortalecimiento del Estado de bienestar, dicha imagen ha podido extraer, asimismo, recursos semánticos de una vieja tradición brasileña de interpretación de su propia historia: la tradición de una mirada maniquea según la cual el mercado sería el reino de las virtudes y el Estado estaría condenado a la eterna repetición de prácticas de corrupción. La fuerza de esa interpretación radica primeramente en su sustrato académico, pues ha sido desarrollada, entre tantos otros lugares, en la obra de dos de los mayores intelectuales del país: en la obra de Sérgio Buarque de Holanda (1995) y, sobre todo, en la obra de Raymundo Faoro (2004), quien alza el concepto de patrimonialismo, derivado no sin polémicas de la obra de Max Weber, a la condición de categoría fundamental de explicación de los problemas de la sociedad brasileña.

Sin embargo, difundida en una versión vulgar en los medios de comunicación y en los círculos seudointelectuales de las clases medias y altas, tal interpretación pudo, de un lado, ayudar a traducir con tonos más brasileños el viejo mantra de la "crítica al populismo" y, de otro, ganar cuerpo en una operación institucional de persecución penal cuyos trámites, resultados directos y desdoblamientos indirectos asombran a cualquier persona que tenga un mínimo de compromiso democrático, incluso uno simplemente liberal. Que el juez que ha tomado una serie de medidas fuertemente cuestionables y que ha impedido fundamentalmente la participación en las elecciones de 2018 de aquel que sería el principal concurrente del presidente de la República entonces elegido (Proner et al., 2017) haya devenido ministro de la justicia y de la seguridad pública del mismo presidente, revela más que todo los compromisos políticos, económicos e ideológicos profundos de la operación "Lava Jato".

No obstante, el Brasil de 2020 no es solo un país que va destruyendo rápidamente la estructura estatal de protección social — que nunca fue muy fuerte-, y cuyos efectos perversos de la retórica del Estado mínimo del "más mercado, menos Estado" se puede ver en el número de muertes relacionadas a la pandemia de la Covid-19. El peso de la narrativa teológico-sacramental ha abierto espacio para una política axiológicamente conservadora. Esta política, a la manera de una paradoja, utiliza el aparato del Estado para intentar retroceder en lo que atañe a las libertades individuales y colectivas logradas con el proceso de efectuación de la Constitución de 1988. Así, las libertades sexuales, la libertad de prensa, la libertad de opinión, la libertad de cátedra o la libertad religiosa, así como los derechos de las mujeres, la población indígena, la población LGBT+ o de la población negra, son a menudo atacadas. 
Pero ese no es el todo del cuadro trágico. Los actos del Gobierno nacional son acompañados de un apoyo y de manifestaciones que simplemente no parecen poder ser explicadas racionalmente. Desde un punto de vista cognitivo, la crítica a las vacunas, la postulación del terraplanismo y la negación de la fuerza o de la existencia de la Covid-19 son ejemplos de esas manifestaciones. A su vez, desde un punto de vista moral son ejemplos la defensa de la tortura, las solicitudes de intervención militar y de reedición del AI-5 (el más duro acto normativo de la dictadura de 1964) y los recientes atentados a profesionales y a protestas en memoria de los muertos por la actual pandemia. El grado de retroceso cognitivo y moral expresado en esas manifestaciones invitan el concepto psicosocial de regresión como categoría explicativa. Esta categoría se refiere a procesos de desaprendizaje profundo en los ámbitos cognitivo y moral (Habermas, 2016b, p. 222; Habermas, 2019, p. 801) que se desencadenan como reacción defensiva a cambios percibidos como amenazas a la integridad de la identidad de cada persona.

La cuestión aquí es que las identidades individuales y colectivas de las clases más altas en Brasil todavía hoy se forman en el molde del esclavismo colonial, más específicamente de la vieja figura del dueño de esclavos. Esa figura tiene como rasgos característicos el patriarcalismo, la misoginia, la cisheteronormatividad, el racismo y la ausencia de límites a su voluntad y a sus órdenes.

A lo largo de las últimas tres décadas, sobre todo en la década de 2000 y en la primera mitad de la década de 2010, tuvieron lugar en Brasil importantes logros en términos de una mayor limitación a la arbitrariedad del poder y de una mayor inclusión social, con más libertad e igualdad: derechos de las mujeres, las personas negras, la población indígena y de la población LGBT+. Para identidades que se desarrollan en el espejo de la imagen del señor colonial — dueño de tierras, cuerpos y vidas—, esos logros no solo representan cambios en la política económica o en el arreglo institucional, sino que representan precisamente amenazas a la integridad de esas identidades. Por ello, para muchas personas, la reacción a los cambios que acompañaron a esos logros no viene articulada solamente, ni primeramente, en discursos pronunciados públicamente o en actuaciones institucionales que se pueden reivindicar públicamente. La reacción a dichos logros y cambios asume la forma de una reacción defensiva, de una regresión que se manifiesta entonces en fenómenos de nítido desaprendizaje cognitivo y moral.

Esa regresión no está restringida a las capas sociales de más poder social y económico. El carácter totalizante del sistema esclavista colonial permanece en su herencia presente: así como hay mujeres que, con sus identidades desarrolladas en ese marco, reproducen rasgos patriarcales, también personas de las clases sociales más bajas incorporan muchas veces como elemento normativo de su autoimagen el perfil de un mando sin límites sobre propiedades, cuerpos y vidas. Por consiguiente, 
no es raro que tales personas igualmente reaccionen de modo defensivo ante los logros y los cambios de los cuales se han podido beneficiar.

Acerca de esa regresión, es importante tener en cuenta los aspectos específicos de la esclavitud negra en Brasil (Freyre, 2003, 2006; Nascimento, 1978; Souza, 2000, 2012). No ha pasado algo semejante en otros países de Sudamérica. Ni siquiera en un país del Caribe como Haití el caso brasileño parece encontrar un equivalente exacto. La persona esclavizada tenía una posición sociológica ambigua: no era solo persona, pero tampoco se la consideraba solo como cosa. De un lado, tomada como cosa, no era posible el entablar profundo, en el cotidiano sociológico, de unas relaciones de reconocimiento recíproco: la sociedad se caracterizaba estructuralmente por una segregación radical, un abismo prácticamente insuperable entre los que tienen mucho y los que nada tienen. Este abismo autorizaba desde las más distintas formas de humillación hasta el uso libre del cuerpo de las personas esclavas, no solo para el trabajo, sino para el sexo no consensuado, para la violencia por puro deleite de los señores y para la muerte dolorosa. De otro lado, como era al mismo tiempo difusamente comprendida como persona en interacciones sociales puntuales - a veces hasta el punto en que se la podía entender como miembro de la compleja noción colonial-patriarcal de familia-, se creaba una situación en que la crítica a la bestialidad de la esclavitud no resonaba con una fuerza suficiente.

Estos dos rasgos, contradictorios y complementarios, siguen dos de los elementos más contundentes del legado esclavista colonial para la sociedad brasileña actual: la necesidad de mantener un abismo entre sus capas más altas y sus capas más bajas —abismo que hace muy difícil, aunque no imposible, una autocomprensión colectiva más generalizada y las prácticas políticas correspondientes_- y la negación ciega de la existencia de problemas sociales muy graves, como el propio racismo, la violencia, la desigualdad abismal, la miseria y el hambre.

Finalmente, otro elemento muy particular viene agregarse para la comprensión, en su diseño fundamental, de la presente crisis brasileña. A diferencia de países como Argentina o Uruguay, en Brasil el proceso de justicia transicional se ha interrumpido drásticamente por lo menos desde 2016, antes de que pudiera resultar en una amplia revisión de la historia oficial y adentrarse en la memoria colectiva. Esta interrupción prematura del proceso de justicia transicional tiene una relación directa con el proceso de regresión y con las identidades que hoy lo viven: para esas identidades, la dictadura de 1964 fue solo un momento más —el más reciente entre aquellos de los que se recuerdan - en que han podido dar rienda suelta a sus fantasías de omnipotencia. Pero, simultáneamente, esa interrupción abre el debate público y las instituciones políticas a una arriesgada, y no suficientemente ponderada, militarización sin gobierno militar que paradójicamente insiste en decirse democrática, mientras amenaza constantemente la democracia misma al pretender tutelarla. 


\section{CONCLUSIONES}

Cuando la crisis objetiva del Estado de bienestar estalló en la superficie de las protestas de 2013 en Brasil, no se podría saber de antemano cuáles decisiones se iban a tomar y cuál dirección se iba a seguir. Pero, al revés, puede afirmarse que las decisiones que desde entonces se han tomado y la dirección que se ha seguido hasta aquí difícilmente habrían tenido lugar sin el punto de partida de la crisis objetiva del intento de conciliación entre capitalismo y democracia.

La tendencia de crisis del estado de bienestar sobrepasa las fronteras del Norte y del Sur. Pero la tendencia de que esa crisis se convierta en una crisis más general y profunda del Estado de derecho como expresión institucional de la democracia es una tendencia típica del Sur global, con la que lamentablemente hemos tenido que acostumbrarnos a convivir en América Latina y el Caribe.

Por ello, en un momento en que nuevamente la crisis objetiva del intento de conciliación entre capitalismo y democracia se manifiesta en distintos países latinoamericanos, la crisis más amplia de Brasil en el año 2020 puede ser pensada como una crisis de nuestra América. Por una parte, la crisis brasileña muestra, como en una caricatura, hasta qué punto la crisis objetiva puede llegar en sus consecuencias. Por otra parte, la centralidad de esa crisis objetiva para la comprensión de la crisis brasileña en su punto de partida invita a reflexionar sobre las posibilidades y los límites del estado de bienestar en Latinoamérica y el Caribe.

En primer lugar, si se toma en serio la constitución y la dinámica sociológica de las sociedades latinoamericanas hoy día — con sus formas de vida plurales, sus planes individuales y colectivos de vida, sus múltiples organizaciones sociales y sus narrativas hegemónicas, pero también con sus movimientos sociales y sus estructuras político-partidarias - no hay otra alternativa concreta en el horizonte próximo sino el estado de bienestar (Habermas, 2015). Esto quiere decir que no tenemos razón alguna para no hacer una defensa fuerte e intransigente del estado de bienestar, aunque esa no sea la defensa de una experiencia concreta cualquiera, como aquella de Europa de la segunda mitad del siglo XX. En otras palabras, no se deben cometer los mismos errores de una reducción sistémica del Estado, sino mantenerlo abierto a los flujos comunicativos — siempre anárquicos en algún grado- de una sociedad civil activa, lo que significa caminar hacia aquello que Habermas (2005a) llama de un "paradigma procedimental del derecho".

Pero como se ha insistido a lo largo de este texto, el estado de bienestar, en su intento de conciliar democracia y capitalismo, tiende ineludiblemente a crisis objetivas: es imposible mantener, al mismo tiempo e indefinidamente, el crecimiento de una economía privada y las políticas de redistribución que buscan ofrecer compensaciones por los efectos colaterales de esa economía, cuyos recursos, en síntesis, se deben 
obtener mediante la tributación de esa misma economía (Habermas, 1992, 2014). Por lo tanto, la defensa del estado de bienestar no puede ser una defensa ingenua.

Así, en segundo lugar, la crisis de Brasil tomada como crisis de nuestra América enseña que no es más posible pensar el modelo de estado de bienestar si se tiene como referencia primordial el Estado nacional. El mercado económico posee una gran habilidad para irse rápidamente con sus flujos de inversión altamente volátiles de un país a otro, tan pronto como el primero no esté dispuesto a ceder a sus presiones ni a atender a sus exigencias. Para hacer frente a un mercado que, desde hace mucho tiempo, ha sobrepasado las fronteras nacionales, es necesario un arreglo políticoinstitucional que también pueda sobrepasar las fronteras (Habermas, 2001, 2005b, 2006, 2012, 2016a). Por ello, la defensa del Estado de bienestar tiene que ser una defensa transnacional (Cabrera, 2009; Cataño y Vásquez, 2005). No se requiere una defensa únicamente desde Brasil, Argentina, Uruguay, Colombia, Chile, Costa Rica o México, sino una defensa latinoamericana y caribeña del estado de bienestar.

Eso no significa renunciar ni abandonar la moldura institucional del Estado nacional. Esta sigue siendo imprescindible por muchas razones, por ejemplo, para expresar historias locales diferentes que no pueden ser simplemente una vez más homogeneizadas violentamente en una especie de macro-Estado. La cuestión no es, pues, el abandono del Estado nacional, sino el fortalecimiento de una actuación común en el nivel de instituciones transnacionales.

Una articulación institucional como esa tendería a obtener logros en el enfrentamiento de las tendencias estructurales de crisis, y podría incluso postergar la crisis. Pero la tendencia en sí misma no sería enteramente bloqueada. Asentada, en última instancia, en la ley del valor del modo de producción capitalista (Marx, 2011, 2013, 2014, 2015), esa tendencia lleva, tarde o temprano, a crisis objetivas mientras el capitalismo no sea desmantelado como sistema económico. Lo que dicha articulación institucional ofrecería entonces no es la promesa, como se ha soñado a lo largo de los "treinta gloriosos" europeos, de extinción de las crisis y de estabilidad eterna de la conciliación entre capitalismo de bienestar y democracia incluyente. Al revés, ella ofrecería la ocasión para un aprendizaje social (Habermas, 2019, pp. 788-807) en un territorio más ancho y en una dimensión temporal más larga, esto es, un aprendizaje justamente sobre la imposibilidad de conciliación duradera entre capitalismo y democracia. Por consiguiente, este aprendizaje sería ante todo acerca de la necesidad de superación del capitalismo para que sea posible la preservación y la profundización del carácter incluyente de la democracia.

Como se ha dicho, cuando la crisis objetiva estalla, pide una decisión, pero no indica la dirección de esa decisión: los caminos que se van a seguir dependen de cómo una sociedad articula, en sus múltiples acciones y discursos, los recursos de sentido que ya están a su disposición, aunque de modo latente. En 2013, la crisis trajo a las calles 
de Brasil sentidos variados, de muchas decisiones posibles. Las primeras protestas fueron una evidente reacción a la apropiación económica privada de bienes públicos, como el transporte colectivo y el propio espacio de las ciudades. Esas protestas eran reflejos del gran aprendizaje de la sociedad brasileña a lo largo de las décadas, principalmente luego de la Constitución de 1988. Pero ese aprendizaje, no obstante su amplitud y sus logros, no ha tenido fuerza para impedir el regreso de las semánticas conservadoras y autoritarias que también salieron a las calles en aquel año, a los puestos de poder y de toma de decisión política. Sin embargo, no tenemos más opción que poner nuestras esperanzas en procesos semejantes —empero más extendidos y robustos- de aprendizajes democráticos.

Si se alcanzara un tal aprendizaje sobre la comprensión no distorsionada de la manera como deseamos vivir y autodeterminar nuestras vidas individuales y colectivas, podríamos volver a pensar en una sociedad poscapitalista sin los errores autoritarios del socialismo real del siglo XX y de estos comienzos del siglo XXI. Podríamos, finalmente, tomar el socialismo como la suma de las condiciones de una libertad en sentidos múltiples, como "el conjunto de condiciones necesarias para formas de vida emancipadas, sobre las que han de empezar entendiéndose los implicados mismos" (Habermas, 1993, p. 107; Habermas, 2005a, p. 60, énfasis del original).

\section{REFERENCIAS}

Arendt, H. (2000). Que é autoridade? En H. Arendt (ed.), Entre o passado e o futuro (M. W. Barbosa, trad. $5^{a}$ ed.) (pp. 127-187). Perspectiva.

Arendt, H. (2006). Da Violência. En H. Arendt (ed.), Crises da República (J. Volkmann, trad.) (pp. 91-156). Perspectiva.

Buitrago, C. (2014). La formación del Estado latinoamericano: entre la dependencia externa y el colonialismo interno (El modelo explicativo y analítico de Marcos Kaplan). Grafía, 11(1), 82-94. http://revistas.fuac.edu.co/index.php/grafia/article/view/509

Cabrera, L. (2009). América Latina y la globalización. Opinión Jurídica, 8(16), 33-46. https://revistas. udem.edu.co/index.php/opinion/article/view/84

Castells, M. (2018). Ruptura: a crise da democracia liberal. Zahar.

Cataño, P. y Vásquez, J. (2005). El fenómeno de la integración subregional a partir del modelo de la Unión Europea y de la Comunidad Andina de Naciones. Opinión Jurídica, 4(8), 39-52. https:// revistas.udem.edu.co/index.php/opinion/article/view/1276

D'eramos, M. (2013). El populismo y la nueva oligarquía. New Left Review (edición en español), 82, 7-40. https://newleftreview.es/issues/82/articles/marco-d-eramo-el-populismo-y-la-nueva-oligarquia.pdf

Dussel, E. (1994). El encobrimiento del outro. Hacia el origen del mito de la modernidade. Ediciones Abya-Yala.

Dussel, E. (1999). Teologia da Libertação - Um panorama do seu desenvolvimento. Editora Vozes.

Dussel, E. (2005). Europa, modernidade e eurocentrismo. Em E. Lander (ed.), A colonialidade do saber: eurocentrismo e ciências sociais. Perspectivas latino-americanas (pp. 24-32). Colección Sur Sur. Clacso. 
Faoro, R. (2004). Os donos do poder: formação do patronato político brasileiro (16 ed.). Globo.

Fernandes, F. (1973). Capitalismo dependente e classes sociais na América Latina. Zahar.

Ferreira, J. (org.). (2010). O populismo e sua história: debate e crítica. Civilização Brasileira.

Freyre, G. (2006). Casa-grande E senzala: formação da família brasileira sob o regime da economia patriarcal (51 ${ }^{a}$ ed). Global.

Freyre, G. (2003). Sobrados e mucambos: decadência do patriarcado rural e desenvolvimento do urbano (14a ed.). Global.

Gomes, D. F. L. (2019). A Constituição de 1824 e o problema da modernidade: o conceito moderno de constituição, a história constitucional brasileira e a teoria da Constituição no Brasil. D’Plácido.

Gomes, D. F. L. (2020). La pérdida de centralidad de la escrita y los riesgos para la democracia: un estudio exploratorio. En G. Scivoletto y C. Cinca (eds.), Desafíos actuales al Estado de Derecho. La legitimidad democrática en cuestión (pp. 1-20). Editores del Sur (en prensa).

Habermas, J. (1992). Legitimation crisis (trad. T. McCarthy). Polity Press.

Habermas, J. (1993). Passado como futuro (trad. F. Siebeneichler). Tempo Brasileiro.

Habermas, J. (2001). The Postnational Constellation - Political Essays (M. Pensky, trad.). The MIT Press.

Habermas, J. (2005a). Facticidad y validez: sobre el derecho y el Estado democrático de derecho en términos de teoría del discurso (trad. M. Jiménez Redondo, $4^{\mathrm{a}}$ ed.). Trotta.

Habermas, J. (2005b). Ciudadanía e identidade nacional. En J. Habermas (ed.), Facticidad y validez: sobre el derecho y el Estado democrático de derecho en términos de teoría del discurso (M. Jiménez Redondo, trad.) (pp. 619-643). Trotta.

Habermas, J. (2006). Entre naturalismo y religión (P. Fabra, D. Gamper, F. Martín, J. L. Lizaga, P. Madrigal y J. C. Velasco, trad.). Paidós Ibérica.

Habermas, J. (2010a). Obras Escolhidas. V. 1, Fundamentação linguística da sociologia (pp. 29-136). Edições 70.

Habermas, J. (2010b). Teoría de la acción comunicativa (M. J. Redondo, trad.). Trotta.

Habermas, J. (2012). Sobre a constituição da Europa (D. Werle, L. Repa y R. Melo, trad.). Unesp.

Habermas, J. (2013). Teoria e práxis: estudos de filosofia social (R. Melo, trad. ). Unesp.

Habermas, J. (2014). Na esteira da tecnocracia. Pequenos escritos políticos XII (L. Repa, trad.). Unesp.

Habermas, J. (2015). A nova obscuridade - A crise do Estado de bem-estar social e o esgotamento das energias utópicas. En J. Habermas, A nova obscuridade. Pequenos escritos políticos V. (L. Repa, trad.) (pp. 209-238). Unesp.

Habermas, J. (2016a). O ocidente dividido (B. Tavolari, trad.). Unesp.

Habermas, J. (2016b). Para a reconstrução do materialismo histórico (R. Melo, trad.). Unesp.

Habermas, J. (2019). Auch eine Geschichte der Philosophie (2 B). Suhrkamp.

Holanda, S. B. (1995). Raízes do Brasil (26a ed.). Companhia das Letras.

Levitsky, S. y Ziblatt, D. (2018). Como as democracias morrem. Zahar.

Marini, R. M. (1971). Subdesarrollo y revolución. Siglo Veintuno Editores. 
Marx, K. (2011). Grundrisse: manuscritos econômicos de 1857-1858: esboços da crítica da economia política (M. Duayer, N. Schneider, A. H. Werner y R. Hoffman, trad.). Boitempo.

Marx, K. (2013). O Capital - Crítica da economia política. L. 1, O processo de produção do capital (R. Enderle, trad.). Boitempo.

Marx, K. (2014). O Capital - Crítica da economia política. L. 2, O processo de circulação do capital (R. Enderle, trad.). Boitempo.

Marx, K. (2015). O Capital - Crítica da economia política. L. 3, O processo global da produção capitalista (R. Enderle, trad.). Boitempo.

Mignolo, W. (2003). Histórias locais/projetos globais: colonialidade, saberes subalternos e pensamento liminar. Editora UFMG.

Mounk, Y. (2019). O povo contra a democracia: porque nossa liberdade corre perigo e como salvá-la. Companhia das Letras.

Nascimento, A. do (1978). O genocídio do negro brasileiro: processo de um racismo mascarado. Paz e Terra.

Panotto, N. (2013). Religión y nuevas formas de militancia: pentecostalismo y política en Capital Federal. Desafíos, 24(61-62), 203-221. https://doi.org/10.12804/desafios26.02.2014.03

Proner, C., Cittadino, G., Ricobom, G., Dornelles, J. R. (ed.) (2017). Comentários a uma sentença anunciada - O processo Lula. Projeto Editorial Práxis.

Quijano, A. (1992). Colonialidad y Modernidad/Racionalidad. En H. Bonilla (ed.), Los conquistados (pp. 437-449). Tercer Mundo Ediciones.

Quijano, A. y Wallerstein, I. (1992, nov.). Americanity as a Concept or the Americas in the Modern World-System. International Social Science Journal, 134, 549-556.

Runciman, D. (2018). Como a democracia chega ao fim. Todavia.

Santos, W. G. (1962). Quem dará o golpe no Brasil? Civilização Brasileira.

Sevaybricker, M. (2019). Democracias no século XXI: causas, sintomas e estratégias para superar a sua crise (manuscrito presentado para publicación). Departamento de Ciências Humanas, Universidade Federal de Lavras.

Souza, J. (2000). A modernização seletiva: uma reinterpretação do dilema brasileiro. UnB.

Souza, J. (2012). A construção social da subcidadania: para uma sociologia política da modernidade periférica. UFMG; Iuperj.

Streeck, W. (2012, mar.). As crises do capitalismo democrático (trad. Alexandre Morales). Novos Estudos, 92, 35-56. http://www.scielo.br/scielo.php?script=sci_arttextEpid=S0101-33002012000100004.

Vilela, D. M. (2013). Utopias esquecidas. Origens da Teologia da Libertação. Fonte Editorial. 